\title{
Anti-HER2/PBD-MA Antibody-drug Conjugate DHES0815A
}

National Cancer Institute

\section{Source}

National Cancer Institute. Anti-HER2/PBD-MA Antibody-drug Conjugate DHES0815A.

NCI Thesaurus. Code C155940.

An antibody-drug conjug ate (ADC) consisting of a monoclonal antibody targeting human epidermal growth factor receptor 2 (ERBB2; EGFR2; HER2) linked to a DNA minor groove crosslinking agent pyrrolo[2,1-c][1,4]benzodiazepine monoamide (PBD-MA), with potential antineoplastic activity. Upon intravenous administration of ADC DHES0815A, the monoclonal antibody moiety targets and binds to HER2 expressed on tumor cells. Following receptor internalization and lysosome-mediated cleavage, the cytotoxic PBD-MA moiety is released. In turn, the imine groups of the PBD-MA moiety bind to and crosslink specific sites of DNA, resulting in DNA strand breaks, cell cycle arrest, and cell death in HER2 expressing tumor cells. HER2, a tyrosine kinase receptor, is overexpressed by many cancer cell types. 